\title{
Cellular response to cetuximab in PTEN-silenced head and neck squamous cell carcinoma cell line
}

\author{
JIHANE MRIOUAH ${ }^{1}$, CEDRIC BOURA $^{1}$, SOPHIE PINEL ${ }^{1}$, ANNE-SOPHIE CHRETIEN $^{1,3}$, \\ ALEXANDRE FIFRE $^{2}$, JEAN-LOUIS MERLIN ${ }^{1,3}$ and BEATRICE FAIVRE ${ }^{1}$
}

\author{
${ }^{1}$ EA 4421 SiGReTO Signalisation, Genomique et Recherche Translationnelle en Oncologie, UHP-Nancy 1, Nancy-Universite, \\ 54505 Vandoeuvre-les-Nancy; ${ }^{2}$ CNRS EAC 7054, Centre de Recherches Chirurgicales Henri-Mondor, \\ Universite Paris XII-Creteil, 94010 Creteil cedex; ${ }^{3}$ Unite de Biologie des Tumeurs, \\ Centre Alexis Vautrin, 54511 Vandoeuvre-les-Nancy, France
}

Received May 25, 2010; Accepted July 12, 2010

\section{DOI: 10.3892/ijo_00000809}

\begin{abstract}
The implication of loss of PTEN expression in resistance to targeted therapy has already been described in many tumor types. The absence of response to anti-EGFR agents in PTEN-deficient tumors relies on persistent activation of signaling pathways downstream of pEGFR. To investigate the role of PTEN loss of expression in head and neck squamous cell carcinoma (HNSCC) response to cetuximab, we used siRNA in Cal 27 cells and then evaluated key signaling protein activation (pAKT and pERK 1/2) as well as cell viability and proliferation. PTEN silencing in Cal 27 cells led to a constitutive activation of signaling pathways evidenced by a strong increase in pAKT and pERK $1 / 2$ expression. Moreover, PTEN-silenced cells did not show any significant changes either in cell viability or proliferation, only slight modifications on cell cycle. Additionally and unpredictably, our results indicated that PTEN silencing, led to a drastic reduction in pEGFR expression whereas total EGFR level did not significantly vary. Strikingly, despite this overactivation of signaling pathways ruling cell survival and proliferation in siPTEN cells, cetuximab fully exerted pAKT and pERK 1/2 inhibition of expression, similarly to its effect in untransfected Cal 27 cells. In conclusion, our study established that in Cal 27 cells, cetuximab keeps full ability to inhibit EGFR-dependent mechanisms, as shown by a decreased pAKT and pERK 1/2 level of expression, despite a strong PTEN silencing-induced overactivation. In Cal 27 cells, loss of PTEN expression does not lead to a loss of cetuximab efficacy in inhibiting EGFRdownstream signaling pathways, contrarily to data shown in previous works conducted in other tumor types.
\end{abstract}

Correspondence to: Dr Beatrice Faivre, EA 4421 SiGReTO, UHP-Nancy 1, Nancy Universite, Faculte de Pharmacie, 5-7 rue Albert Lebrun, BP 80401, 54001 Nancy cedex, France

E-mail: beatrice.faivre@pharma.uhp-nancy.fr

Key words: head and neck squamous cell carcinoma, pEGFR, cetuximab, PTEN, AKT, ERK 1/2, $\mathrm{G}_{2} \mathrm{M}$ phase

\section{Introduction}

For the last decade, EGFR (epidermal growth factor receptor) has been extensively investigated because of its key role in cancer biology $(1,2)$. EGFR is implicated in cell proliferation, differentiation and growth, as well as angiogenesis and cell adhesion (3). EGFR activation by ligand binding induces dimerization then autophosphorylation of the receptor, and activates several signaling pathways among which, PI3K/AKT and MAPK pathways play a major role (1). EGFR is overexpressed in many human malignancies including colorectal cancer, breast cancer, and lung cancer. In head and neck squamous cell carcinoma (HNSCC), EGFR overexpression has been detected in $90-100 \%$ of tumor specimens and has been correlated to a worse prognosis $(4,5)$. Based on this, anti-EGFR targeted therapies have been proposed to prevent EGFR activation and its downstream signaling pathways. Monoclonal antibodies, such as cetuximab, directed against the extracellular portion of EGFR, have been reported to reduce proliferation and enhance apoptosis in cancer cells (5-7).

Nevertheless, since cetuximab has been approved by the FDA (Food and Drug Administration) in 2003, cases of resistance to cetuximab have been progressively reported. Notably in HNSCC patients, Cohen (8) reported modest response rates $(5-15 \%)$ to cetuximab depending on dose and schedule. In this work, the author underlined the lack of identification of subgroups of patients that could respond or benefit of the use of targeted agents. Predictive markers for response to targeted therapies are needed and prospected in persistent activation of the PI3K/AKT and MAPK signaling proteins, which, have been related to loss of efficacy of anti-EGFR therapies in HNSCC as well as in other tumor types (9-12).

$K R A S$ activating mutations are validated as a predictive marker for no response to cetuximab in metastatic colorectal cancer $(13,14)$. However, wild-type KRAS patients do not necessarily benefit from such treatment (15), demonstrating thus, that determining KRAS status is not sufficient to ensure cetuximab treatment efficiency.

PI3K/AKT pathway constitutive activation has also been frequently pointed out in mechanisms of resistance to targeted therapies. As the main regulator of PI3K/AKT pathway activation, the key role of PTEN phosphatase has been high- 
lighted in resistance towards anti-HER therapies in non-small cell lung cancer $(16)$, breast cancer $(17,18)$, colorectal cancer $(19,20)$, and prostate cancer cells (21). PTEN is one of the most mutated gene in human cancers $(22,23)$. PTEN antagonizes PI3K, preventing then PDK1-mediated activation of AKT via $\mathrm{PiP}_{3}$. An indirect PTEN regulation on MAPK pathway has also been established, by inhibiting Shc-mediated Ras activation (24). Hence, PTEN modulation of PI3K/AKT and MAPK pathways is critical to impair cell survival and proliferation. PTEN has also a direct implication in limitation of cell cycle progression from $G_{0} / G_{1}$ to $S(25)$.

Therefore, loss of PTEN expression, leads to persistent activation of signaling proteins and provides a sizeable advantage to cells in term of survival and proliferation. In HNSCC tumors, loss of PTEN expression occurs in 10-30\% of the cases $(23,26)$. Squarize et al $(27)$ showed a correlation between PTEN expression and histological malignancy: PTEN was expressed in adjacent non-tumor epithelium whereas tumors with a high score of malignancy did not express PTEN. Despite the observations made about loss of PTEN expression, there is no evidence of its eventual role in modest efficacy of cetuximab monotherapy in HNSCC.

The aim of our work was to investigate whether PTEN loss of expression has an impact on HNSCC cells sensitivity to cetuximab, regarding its impact on EGFR downstream signaling pathways. Using siRNA transfection in Cal 27 human HNSCC cell line, we establish that loss of PTEN expression induces as expected, a strong upregulation of pAKT and pERK 1/2 but does not play a determinant role in Cal 27 cells in cetuximab sensitivity. Strikingly our results suggest that persistent activation of EGFR signaling pathways induced by PTEN silencing does not lead to a loss of molecular activity of cetuximab.

\section{Materials and methods}

\section{Cal 27 cell line and chemicals}

Cell culture. Cal 27, a human head and neck squamous cell carcinoma (HNSCC) cell line derived from a tongue squamous cell carcinoma isolated from a patient prior to treatments, was obtained from Dr J.L. Fischel (Centre Antoine Lacassagne, Nice, France) (CRL-2095 ${ }^{\mathrm{TM}}$, ATCC). The cell line was cultured in phenol-red free RPMI-1640 (Invitrogen) supplemented with $10 \%$ of heat-inactivated fetal calf serum (FCS, Sigma Aldrich, France), 2 mM of L-glutamine, $100 \mathrm{U} / \mathrm{ml}$ penicillin and $100 \mu \mathrm{g} / \mathrm{ml}$ streptomycin at $37^{\circ} \mathrm{C}$ in a humidified atmosphere of $5 \% \mathrm{CO}_{2}$ in air.

KRAS genotyping. Cal 27 cells were checked for KRAS mutations (codons 12 and 13) using two-step PCR-RFLP (for restriction fragments length polymorphism) according to Schimanski et al (28). Briefly, DNA was extracted from $10^{9}$ cells suspension. The amount of DNA isolated was determined spectrometrically and $100 \mathrm{ng}$ was used as template for the first PCR (Master Cycler Gradient, Eppendorf, Germany) with the oligonucleotide primers Ras A (sense 5'-ACTGAATAT AAACTTGTGGTCCATGGAGCT-3') and Ras B (antisense 5'-TTATCTGTATCAAAGAATGGTCCTGCACCA-3'). PCR products were then submitted to enzymatic digestion with either BstXI or XcmI, restricting the amplicon if the first two bases of codon 12 (BstXI) and codon 13 (XcmI) were wild-type. The first digest $(2 \mu 1)$ was used as template for the second PCR in which primer Ras C (antisense 5'-GGATG GTCCTCCACCAGTAATATGGATATTA-3') was used instead of Ras B. Second PCR (7 $\mu 1)$ was digested with either Bst XI or XcmI. The digest $(10 \mu 1)$ was submitted to PAGE stained with ethidium bromide and analysed under UV light (GelDoc EQ, Bio-Rad). DNA extracts from cell lines bearing codon 12 or/and 13 mutations were added as positive controls.

Chemicals. Cetuximab (Erbitux ${ }^{\mathrm{TM}}$ ), a chimeric monoclonal antibody targeting EGFR (Merck Serono, Lyon, France) and was diluted to the final concentration of $20 \mu \mathrm{g} / \mathrm{ml}$ in fresh medium containing $2 \%$ FCS before each experiment. Cetuximab concentrations and duration of exposure have been determined from previous experiments performed on three different HNSCC cell lines (FaDu, Cal 33, Cal 27). Culture medium was replaced by cetuximab solution at day 5 following cell seeding.

\section{PTEN silencing}

Design and construction of small interfering RNA of PTEN. Based on human PTEN mRNA sequence (accession no. NM 000314), specific siRNA duplexes designed, synthesized and annealed by Eurogentec (Seraing, Belgium) were evaluated and the duplex leading to the highest silencing of PTEN expression was selected. During our experiments, we have tested five duplexes targeting different regions of PTEN mRNA then selected one for the subsequent study. The selected RNA duplex is defined as sense: 5'-GCUACCUGUUAAAGAA UCA-3' and antisense: 5'-UGAUUCUUUAACAGGUAG-3'. The negative control siRNA sequence was developed by Eurogentec and has no significant homology to any known human gene sequence.

SiRNA transfection. Cal 27 cells were plated at day 0 in 6-well plates at $8 \times 10^{4}$ cells per well in $2 \mathrm{ml}$ phenol-red free supplemented RPMI-1640 medium and allowed to grow. When cells were $60 \%$ confluent (day 3 ), the culture medium was replaced by a FCS-free RPMI-1640 medium. Each well received then $10 \mathrm{nM}$ siRNA by using INTERFERin ${ }^{\mathrm{TM}}$ according to the manufacturer's recommendations (PolyPlus Transfection, Illkirch, France). After 4 h, the transfection was stopped by replacing serum-free RPMI-1640 medium with 10\% FCS-RPMI-1640 medium.

The transient PTEN-deficient cells (defined as 'SiPTEN cells') and cells transfected by the control sequence (defined as 'siRNA neg. cells') were expanded and ultimately used to monitor PTEN protein expression, 48, 72, 96 and $120 \mathrm{~h}$ of transfection by Western blot analysis. The expression of phosphoproteins among EGFR signaling pathways (pEGFR, pAKT, pERK 1/2) was quantified using Bio-plex ${ }^{\circledR}$ protein array (BPA) technology as described previously (29) and checked by Western blot analysis while PTEN expression was inhibited.

\section{Protein assays}

Proteins extraction. Proteins were extracted from monolayered cell cultures using a cell lysis kit (Bio-Rad, MarnesLa-Coquette, France) according to the manufacturer's recom- 
mendations. Briefly, cell cultures were exposed for $15 \mathrm{~min}$ to the lysis solution containing phenylmethylsulfonyl fluoride antiprotease. After centrifugation at $1200 \mathrm{~g}$ at $4^{\circ} \mathrm{C}$, soluble proteins from supernatant were stored frozen at $-80^{\circ} \mathrm{C}$ until used. Protein analyses were then performed using Western blot analysis and BPA.

Western blot analysis. Protein aliquots $(6 \mu \mathrm{g})$ were denatured in the Laemmli buffer, containing $B$ mercaptoethanol and by heating for $5 \mathrm{~min}$ at $95^{\circ} \mathrm{C}$, before to be resolved in SDS-polyacrylamide gel electrophoresis. The separated proteins were transferred using a Mini TransBlot cell onto PVDF membranes (Amersham Biosciences, Orsay, France). To avoid non-specific binding, the membranes were blocked by 1-h incubation in Tris base-buffered saline prepared with $0.1 \%(\mathrm{v} / \mathrm{v})$ Tween-20 (TBST buffer) containing $5 \%(\mathrm{w} / \mathrm{v})$ bovine serum albumin. The PVDF membranes were then allowed to react overnight at $4^{\circ} \mathrm{C}$ with the primary antibodies diluted in TBST buffer containing $5 \%$ bovine serum albumin as follows: mouse antibodies against human PTEN (Monoclonal 3627, Dako, 1:1000 dilution), against phospho-EGFR (no. 2236, Cell Signaling 1:1000 dilution), phospho-ERK 1/2 (no. 9106, Cell Signaling, 1:1000 dilution), and rabbit antibodies against phospho-AKT (no. 9271, Cell Signaling, 1:1000 dilution), and B-tubulin (no. 9104; Santa Cruz, 1:3000 dilution) were used as the internal control.

After being washed with TBST buffer, the membranes were incubated for $1 \mathrm{~h}$ at room temperature with specific horseradish-peroxidase-secondary anti-IgG1 polyclonal antibodies (Santa Cruz Biotechnology, Heidelberg, Germany). Subsequently, immunoreactive proteins were visualized using the enhanced chemiluminescence procedure (Amersham). Quantification of relative band densities was performed using standard densitometry scanning techniques (Rasband, WS, ImageJ, US National Institute of Health, Bethesda, MD).

Bio-Plex $x^{\circledR}$ phosphoprotein array. Phosphoprotein expression was analyzed using Bio-Plex protein array (30) based on multiplex sandwich bead immunoassays. Phosphoprotein determination kits (Bio-Rad) were used according to the manufacturer's recommendations. Briefly, protein extracts (6 $\mu \mathrm{g}$ in $25 \mu \mathrm{l})$ were transferred into 96-well dishes and diluted with $25 \mu \mathrm{l}$ buffered solution (assay buffer, Phosphoprotein Reagent kit, Bio-Rad). Fluorescent capturing beads coupled to antibodies (Bio-Plex phosphoprotein assays, Bio-Rad) directed against p-EGFR or downstream signaling phosphoproteins (p-AKT, p-ERK 1/2) were mixed, and added into each well and incubated overnight at $20^{\circ} \mathrm{C}$. The plates were then washed (Bio-Plex phosphoprotein assays, Bio-Rad) and incubated for $30 \mathrm{~min}$ at room temperature with the detection biotinylated antibodies under shaking, fixing each target protein. The streptavidin-phycoerythrin solution (Bio-Plex phosphoprotein assays, Bio-Rad) was then added for $10 \mathrm{~min}$ at room temperature. The analysis consisted in a double laser fluorescence detection allowing simultaneous identification of the target protein through the red fluorescence emission signal of the bead and quantification of the target phosphoprotein expression through the fluorescence intensity of phycoerythrin. Results were recorded as fluorescence intensities and compared to positive controls. Positive controls consisting in standard protein extract from cell lines were added to each series.

\section{Cell viability and proliferation}

Metabolic activity. Cellular metabolic activity was determined by monitoring the mitochondrial activity using 3-(4,5 dimethylthiazol-2-yl)-2,5-diphenyl-tetrazolium bromide assay (MTT, Sigma). Briefly, $2 \times 10^{4}$ cells were plated in $1 \mathrm{ml} /$ well of $10 \%$ FCS-RPMI-1640 into 24-well dishes. At day 5 (2 days after transfection), the culture medium was replaced by $2 \%$ FCSRPMI-1640 medium containing 0 or $20 \mu \mathrm{g} / \mathrm{ml}$ cetuximab for $48 \mathrm{~h}$.

After this incubation period, $0.5 \mu \mathrm{mol} / 1 \mathrm{MTT}$ were added into each well. Following a 2-h incubation at $37^{\circ} \mathrm{C}$, formazan crystals resulting from MTT metabolization were solubilized and cells were lysed by adding $400 \mu 1$ of DMSO into each well. The absorbance of the resulting solution was measured at $570 \mathrm{~nm}$ using wells without cells as blank (Multiskan Ascent microplate photometer, Thermo Scientific, Gometz-Le-châtel, France).

Proliferation assays. For trypan-blue exclusion assay, cells were cultivated in a 6-well dishes and exposed to cetuximab following PTEN silencing (as mentioned above). Cells were detached using a $5 \mathrm{X}$-trypsin-EDTA solution (Sigma) for $7 \mathrm{~min}$ at $37^{\circ} \mathrm{C}$ and resuspended in a complete medium. Cells were then stained with $0.4 \%$ trypan-blue solution and viable cells were counted twice using a Malassez hemocytometer.

Cell cycle analysis. The effects of cetuximab and of the loss of PTEN silencing on cell proliferation were determined using flow cytometry analysis of cell cycle progression. Either transfected or untransfected Cal 27 cells were washed twice with calcium magnesium-free-PBS (CMF-PBS) before being trypsinized and harvested. The cells were then pelleted at $200 \mathrm{~g}$ for $10 \mathrm{~min}$ and fixed in $70 \%$ ethanol (v/v) and stored at $4^{\circ} \mathrm{C}$ until analyzed.

Before analysis, all samples were washed twice and resuspended in CMF-PBS. DNA was stained at room temperature with $1 \mathrm{mg} / \mathrm{ml}$ propidium iodide (Molecular Probes, Invitrogen, Cergy-Pontoise, France) in the presence of $10 \mu \mathrm{g} / \mathrm{ml}$ of RNase A (Sigma, St. Louis, MO). DNA content was determined by Fluorescence-Activated Cell Sorting (FACS) analysis using flow cytometry FACS-Calibur (Becton-Dickinson, Le Pont de Claix, France). Data from at least 10,000 events were analyzed using ModFit LT software (Verity Software House, Topsham, ME, USA).

Statistical analysis. All experiments were performed at least in triplicate and the results are presented as mean values \pm standard deviation. Non-parametric Mann-Whitney test was employed to determine the statistical significance with a limit set to $\mathrm{P}<0.05$ using Statview 5.0 software (SAS Institute Inc., USA).

\section{Results}

Cal 27 cells sensitivity to cetuximab. Cetuximab effect on cell viability of HNSCC cell lines was first investigated in FaDu (HTB-43), Cal 27 (CRL-2095) and Cal 33. Our results 


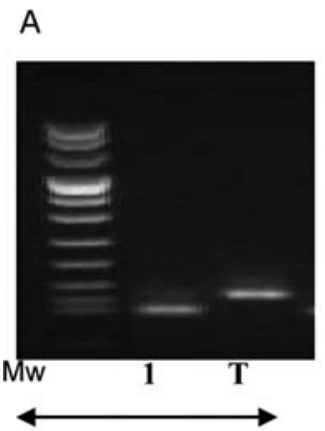

Dig. Bst XI (codon 12)

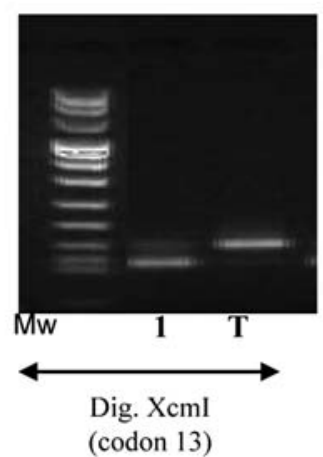

(codon 13)

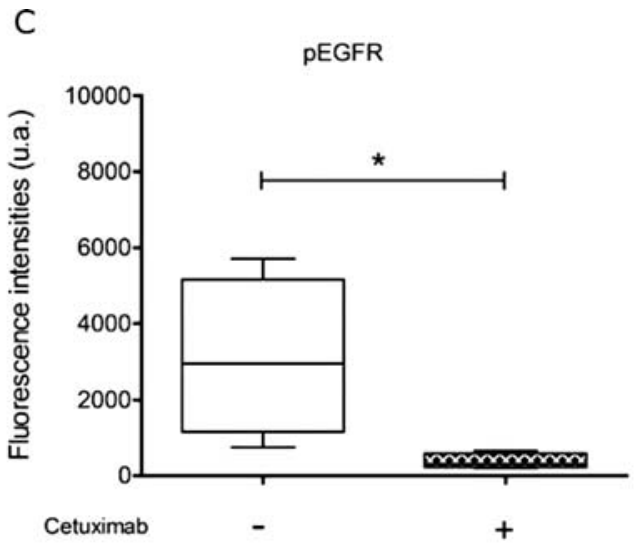

PAKT

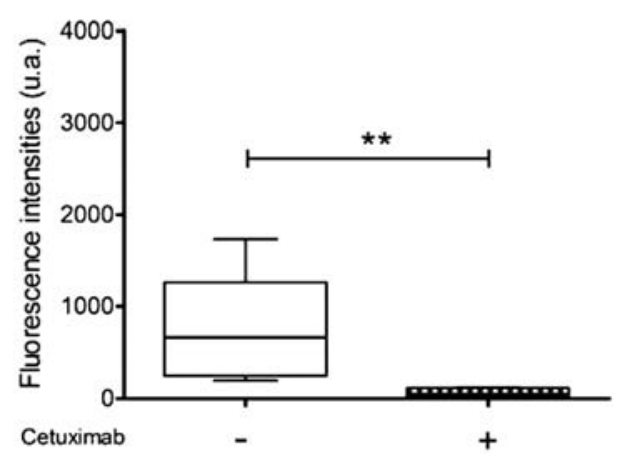

pERK $1 / 2$

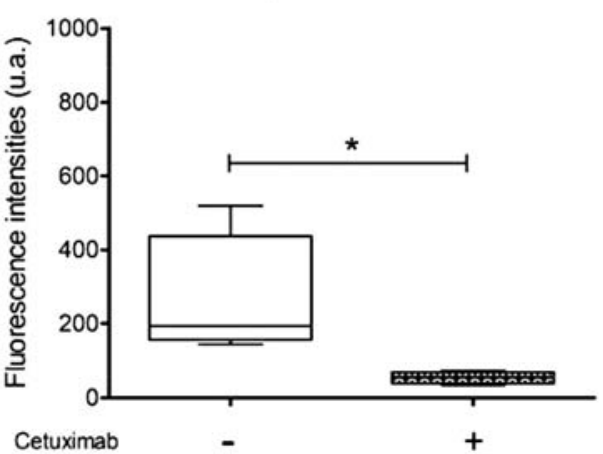

Figure 1. Signaling proteins status of expression in Cal 27 cells. Cal 27 cells were found non-mutated for KRAS using PCR-RFLP. (A) Agarose gel loaded with products from PCR-RFLP: DNA extracts from Cal 27 (line 1) cells line were submitted to double PCR amplification after BstXI and XcmI enzymatic digestions allowing discrimination of codons 12 and 13 mutations. Codons 12 and 13-mutated cell lines were used as positive controls (line T). Cetuximab induced a strong inhibition on EGFR signaling phosphoproteins of Cal 27 cells. Proteins extracts were collected from cells exposed, or not, to cetuximab $(20 \mu \mathrm{g} / \mathrm{ml})$ for $48 \mathrm{~h}$. Phosphoprotein expression was investigated by Western blotting using specific antibodies. $\beta$ tubulin was used as control for the equal loading of proteins (B). Levels of pEGFR, pAKT and pERK 1/2 expression were also determined by Bioplex Protein Array assay and are presented as fluorescence intensities measured in protein extracts. The same amount of proteins was used in all BPA experiments. ${ }^{*} \mathrm{P}<0.05$, ${ }^{* *} \mathrm{P}<0.01$, versus untreated control cells (C).

established that FaDu cell line was resistant to cetuximab. Conversely Cal 27 and Cal 33 cell lines were found sensitive (data not shown). As the aim of the work was to identify the role of PTEN protein in cells sensitivity to cetuximab, the most sensitive cell line was chosen for all further experiments. Moreover, there is no PTEN-deficient HNSCC cell line available, so we used the siRNA directed against PTEN mRNA in Cal 27 cells.

Cal 27 cells were characterized for KRAS status, and PTEN, EGFR and downstream signaling phosphoprotein expression. Using two-step PCR-RFLP (restriction fragments length polymorphism), we determined that Cal 27 cells were KRAS wild-type (Fig. 1A). Then, using Western blotting and Bioplex protein assay (BPA) arrays, Cal 27 cells were found to express basal pEGFR, PTEN and phosphoprotein levels (Fig. 1B and C).

Exposure to cetuximab induced a $90 \%$-inhibition of pEGFR expression, leading to the subsequent downregulation of pAKT (93\%) and pERK 1/2 (71\%) (Fig. 1B and C). As a consequence, $\mathrm{Cal} 27$ cells were found sensitive to cetuximab as cell viability was reduced by $20 \%$ (Fig. 2A) and cells number was reduced by $31 \%$ (Fig. 2B). This can be linked to 
A

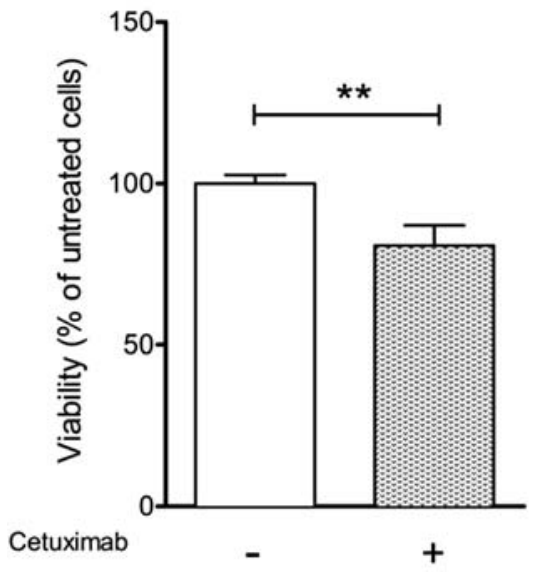

B

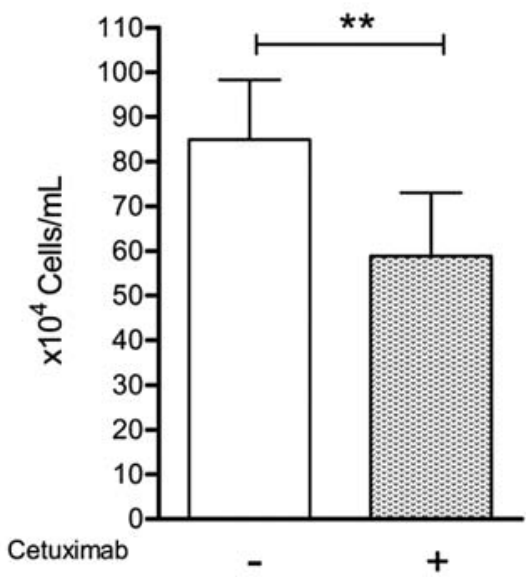

Figure 2. Effect of cetuximab on cells viability and proliferation of Cal 27 cells. Cells were exposed, or not to cetuximab (20 $\mu \mathrm{g} / \mathrm{ml})$, for $48 \mathrm{~h}$ in $2 \%$ FCS RPMI-1640 medium. Mitochondria metabolic activity was measured by MTT tests and reported to the untreated controls. (A). Cal 27 cells proliferation was evaluated by trypan-blue exlusion assays and presented as concentration (cells number x 104/ml). Each sample was stained with $0.4 \%$ trypan-blue solution and counted twice (B). Data are means $\pm \mathrm{SE}$ of at least 3 experiments; ${ }^{* *} \mathrm{P}<0.01$ versus untreated control cells.

Table I. Effect of cetuximab treatment on cell cycle distribution. ${ }^{a}$

\begin{tabular}{lcc}
\hline & \multicolumn{2}{c}{ Cell cycle distribution $(\%)$} \\
\cline { 2 - 3 } Cetuximab & - & + \\
\hline $\mathrm{G}_{0} / \mathrm{G}_{1}$ & $69.4 \pm 3.3$ & $80.9 \pm 4.7^{\mathrm{b}}$ \\
$\mathrm{S}$ & $16.3 \pm 3.6$ & $7.1 \pm 1.5^{\mathrm{b}}$ \\
$\mathrm{G}_{2} / \mathrm{M}$ & $14.4 \pm 2.5$ & $12.0 \pm 4.3$ \\
\hline
\end{tabular}

${ }^{\mathrm{a} C e l l s ~ e x p o s e d ~ o r ~ n o t ~ t o ~ c e t u x i m a b ~}(20 \mu \mathrm{g} / \mathrm{ml})$ for $24 \mathrm{~h}$ were incubated with propidium iodide. Cell cycle was analyzed by flow cytometry and proportion of cells in each phase of cell cycle was calculated using ModFit software and was expressed as percentage. Cetuximabuntreated cells versus cetuximab-treated cells ${ }^{\mathrm{b}} \mathrm{P}<0.01$.

the cetuximab-induced cell cycle perturbations shown by a significant increase $(11.5 \%$; Table $\mathrm{I}, \mathrm{P}<0.01)$ in $\mathrm{G}_{0} / \mathrm{G}_{1}$-phase fraction, leading to depletion in $\mathrm{S}$-phase fraction. Taken together, these results confirmed the ability of cetuximab in Cal 27 cells to block EGFR and downstream signaling activation, and to produce a significant inhibition in proliferation.

Efficacy of PTEN silencing in Cal 27 cells. As expected, PTEN silencing by siRNA sequence delivery through a non-viral transfection was shown to be a progressive and transient phenomenon. To assess silencing efficacy, PTEN protein expression was evaluated by Western blot analyses (Fig. 3). Downregulation of PTEN expression started $48 \mathrm{~h}$ after transfection with a significant $(\mathrm{P}<0.01) 55 \pm 4 \%$ reduction of PTEN expression as compared to siRNA neg. cells. Maximal PTEN silencing was found to reach $90 \pm 6 \%(\mathrm{P}<0.001), 72$ and $96 \mathrm{~h}$ after transfection. Then, PTEN expression was detected again $120 \mathrm{~h}$ after transfection at $25 \pm 6 \%$ of siRNA

\section{Cal $27 \stackrel{\text { SiRNA }}{\text { Neg. SiPTEN }}$}

$48 \mathrm{~h}$

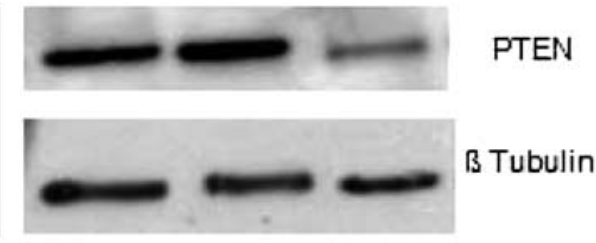

$72 \mathrm{~h}$
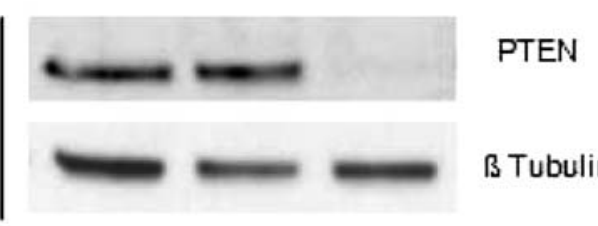

PTEN

B Tubulin

$96 \mathrm{~h}$

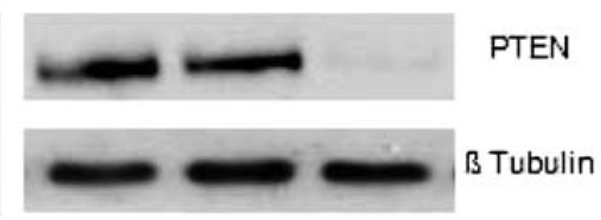

$120 \mathrm{~h}$

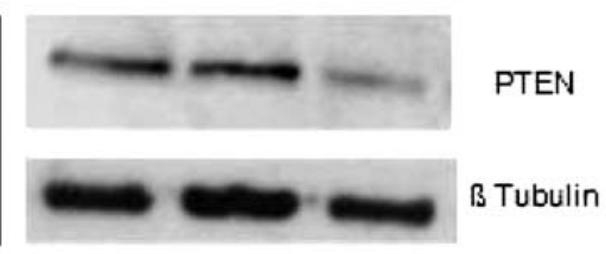

Figure 3. Efficacy of PTEN silencing by siRNA in Cal 27 cells. Cells were transfected with siRNA targeting PTEN or siRNA negative control. Total proteins were extracted every day after transfection $(48,72,96$ and $120 \mathrm{~h}$ ) and subjected to Western blot analysis with the specific antibodies against PTEN.

neg. cells. Based on these results, further experiments in siPTEN cells were scheduled during the time period of maximal PTEN silencing (48-96 $\mathrm{h}$ after transfection). No significant difference was found between untransfected Cal 27 and siRNA neg. cells either in signaling pathways or cell proliferation (numeration, cell cycle distribution) and cell 
A

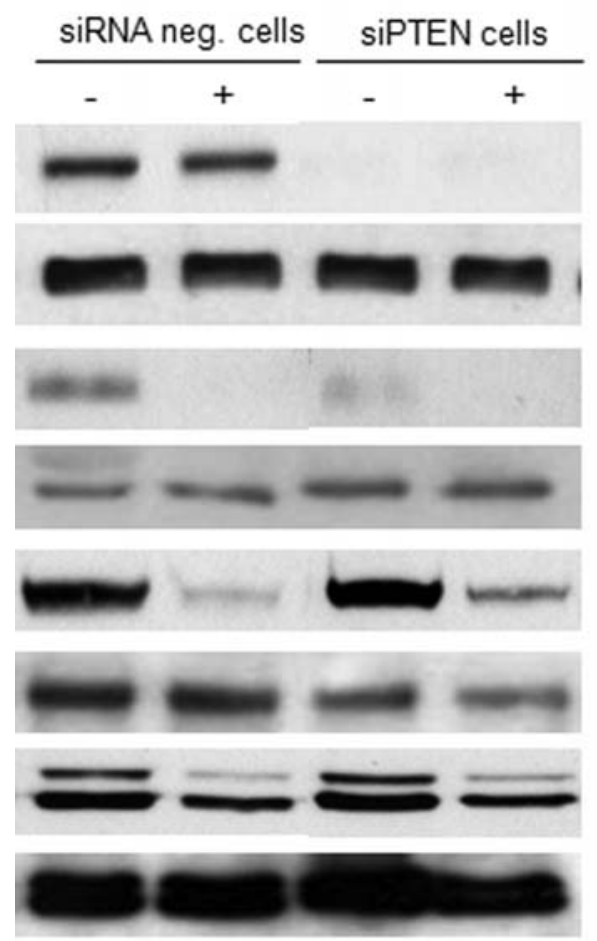

B

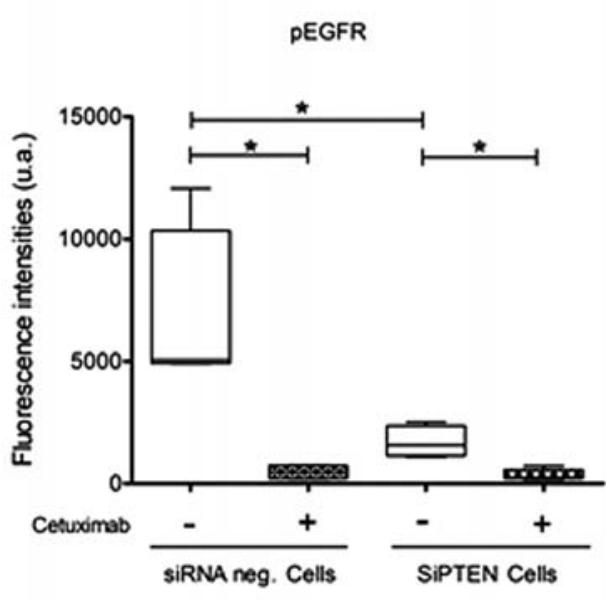

AKT

pERK 1,2

ERK 1,2
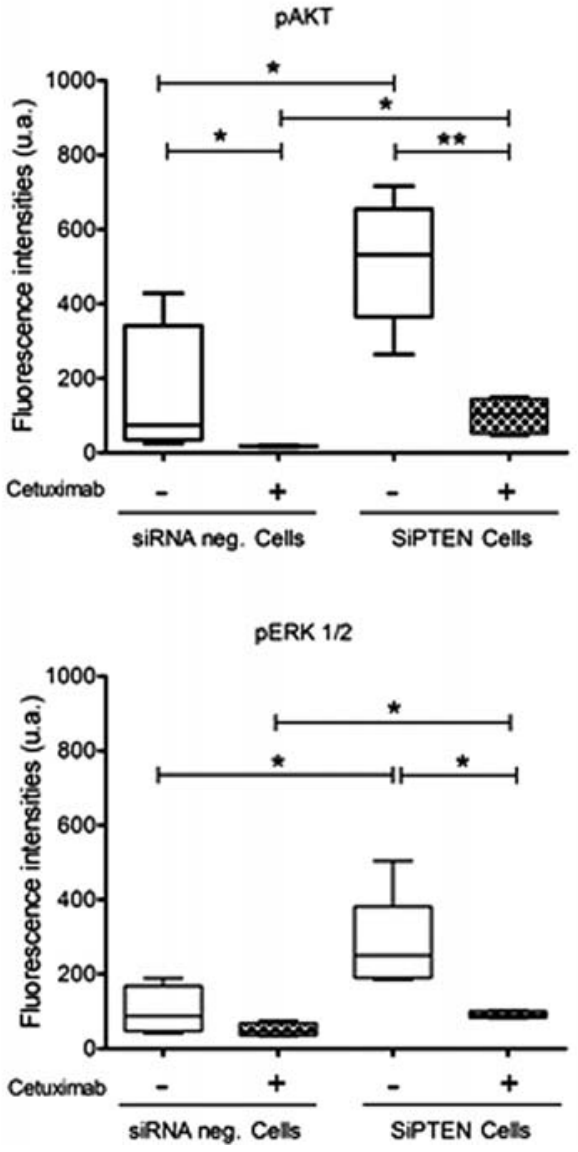

Figure 4. Effect of cetuximab and PTEN silencing on pEGFR and downstream phosphoproteins. Protein extracts were collected $96 \mathrm{~h}$ after transfection either by negative control sequence or PTEN-targeting siRNA, cells were exposed, or not, to cetuximab $(20 \mu \mathrm{g} / \mathrm{ml})$ for $48 \mathrm{~h}$. Phosphoprotein expression was investigated by Western blotting (A), using specific antibodies. $\beta$ tubulin expression was used as control for equal loading of proteins. Expression of pEGFR, pAKT and pERK 1/2 are also presented as fluorescence intensities measured by BPA assay (B). The same amount of proteins $(6 \mu \mathrm{g})$ was used in each BPA experiment. ${ }^{*} \mathrm{P}<0.05 ;{ }^{* *} \mathrm{P}<0.01$. viability. Consequently, every further comparative analysis was performed using siRNA neg. cells as reference.

Impact of PTEN silencing and cetuximab on signaling pathway activation. As expected, $96 \mathrm{~h}$ after transfection, significant and important increases in pAKT (7-fold) and pERK 1/2 (3-fold) expression are shown ( $\mathrm{P}<0.05$, Fig. 4B) in siPTEN cells as compared to siRNA neg. cells. This result indicates the signaling pathway overactivation while PTEN inhibition of expression is maximal. Surprisingly, pEGFR expression was importantly downregulated in siPTEN cells (61\% inhibition) contrarily to total EGFR expression, which was found constant (Fig. 4A). In siPTEN cells, the overactivation of pAKT and pERK $1 / 2$ occurred in this context of pEGFR downregulation, demonstrating thus the constitutive activation of signaling pathways.

Unpredictably, and despite the pEGFR downregulation, cetuximab exposure led to an efficient inhibition of AKT and ERK $1 / 2$ activation in siPTEN cells, to an even greater extent than its effect in siRNA neg. cells. Indeed, in siRNA neg. cells, pEGFR expression was inhibited by $58 \%$ (Fig. 4B) after cetuximab exposure and pAKT and pERK $1 / 2$ expression was inhibited by 74 and $48 \%$, respectively. In siPTEN cells, pEGFR expression was found to be reduced by $85 \%(\mathrm{P}<0.05$, 
A

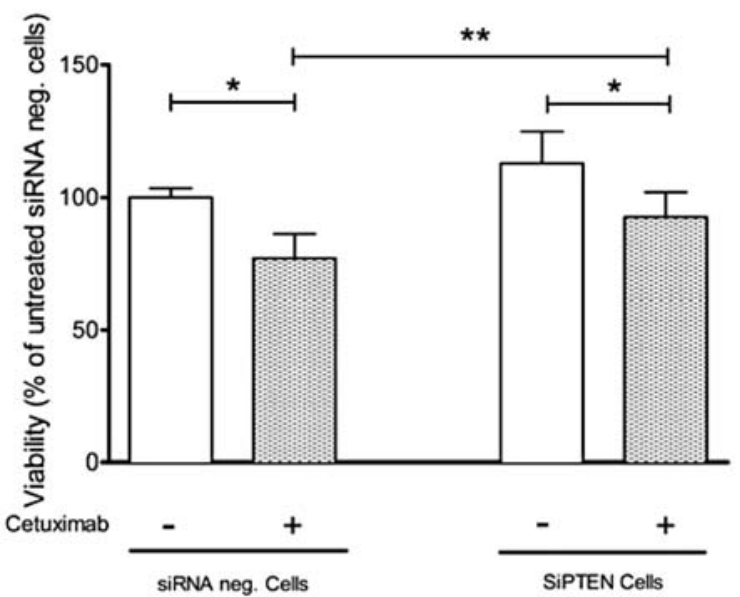

B

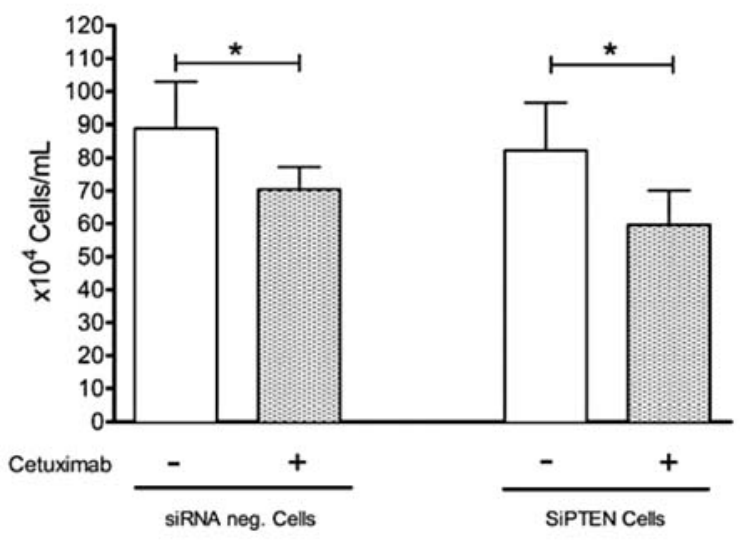

Figure 5. Effect of cetuximab and PTEN silencing on Cal 27 cell viability and proliferation. Forty-eight hours after transfection with either negative control or PTEN siRNA, cells were exposed, or not, to cetuximab $(20 \mu \mathrm{g} / \mathrm{ml})$ for $48 \mathrm{~h}$ in $2 \%$ FCS RPMI-1640 medium. Cell viability was measured by MTT test and compared to the untreated controls (A). For cell numeration, each sample was stained with $0.4 \%$ trypan-blue solution and counted twice (B). Data are the mean \pm $\mathrm{SE}$ of at least 3 experiments; ${ }^{*} \mathrm{P}<0.05 ;{ }^{* *} \mathrm{P}<0.01$.

Table II. Effect of cetuximab treatment and PTEN silencing on cell cycle distribution. ${ }^{a}$

\begin{tabular}{|c|c|c|c|c|}
\hline \multirow{3}{*}{ Cetuximab } & \multicolumn{4}{|c|}{ Cell cycle distribution (\%) } \\
\hline & \multicolumn{2}{|c|}{-} & \multicolumn{2}{|c|}{+} \\
\hline & SiRNA control cells & SiPTEN control cells & SiRNA control cells & SiPTEN cells \\
\hline $\mathrm{G}_{0} / \mathrm{G}_{1}$ & $70.3 \pm 2.7$ & $66.0 \pm 2.0^{\mathrm{c}}$ & $77.7 \pm 4.0^{\mathrm{b}}$ & $73.8 \pm 5.2^{\mathrm{b}}$ \\
\hline S & $15.9 \pm 2.9$ & $16.1 \pm 3.3$ & $8.2 \pm 1.8^{b}$ & $8.0 \pm 2.5^{\mathrm{b}}$ \\
\hline $\mathrm{G}_{2} / \mathrm{M}$ & $13.8 \pm 3.5$ & $17.9 \pm 2.6^{\mathrm{c}}$ & $14.1 \pm 3.2$ & $18.2 \pm 1.0$ \\
\hline
\end{tabular}

${ }^{a}$ Cells exposed or not to cetuximab for $24 \mathrm{~h}$ were incubated with propidium iodide. Cell cycle was analyzed by flow cytometry. Proportion of cells in each phase of cell cycle was calculated using ModFit software and was expressed as percentage. Cetuximab-untreated cells versus cetuximab-treated cells: ${ }^{b} \mathrm{P}<0.05$. SiPTEN cells versus siRNA control cells: ${ }^{\mathrm{c}} \mathrm{P}<0.05$.

Fig. 4B), consequently pAKT and pERK $1 / 2$ expression was significantly decreased by 81 and by $70 \%$, respectively $(\mathrm{P}<0.05$, Fig. 4B). Even though cetuximab was efficient in blocking signaling pathway activation in siPTEN cells, there was a slight residual level of the phosphoprotein expression. Signaling pathway activation in siPTEN cells exposed to cetuximab was then stronger than in siRNA neg. cells unexposed to cetuximab $(\mathrm{P}<0.05)$ and that, independently of pEGFR, which, was equally inhibited in siPTEN, and siRNA neg. cells. Strikingly, these results established that the PTEN silencing-mediated constitutive activation of signaling pathways in Cal 27 cells, can be efficiently overcome by cetuximab exposure.

Impact of PTEN silencing and cetuximab on cell viability and proliferation. PTEN silencing did not induce any significant

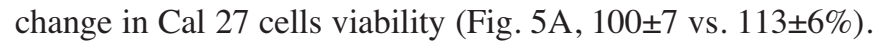
Cetuximab exposure induced a decrease of $20 \pm 3 \%(\mathrm{P}<0.05)$ in cells viability (Fig. 5A) in each case. Thus, no significant difference in cetuximab effect on cell viability was found depending on PTEN status in Cal 27 cells. However, as siPTEN cells were found to express residual pAKT and pERK 1/2 despite cetuximab exposure, cell viability of siPTEN cells was maintained at $93 \%$ of siRNA neg. cells not exposed to cetuximab (considered as the $100 \%$ viability).

Unexpectedly, PTEN silencing did not produce any impact in Cal 27 cell proliferation as no significant difference was found between the number of siRNA neg. cells and siPTEN cells $(\mathrm{P}>0.2)$. Similarly, cetuximab exposure led to the same decrease in proliferation of siRNA neg. cells and siPTEN cells, as the cell number was reduced by $25 \pm 5$ and $20 \pm 2 \%$ respectively $(\mathrm{P}>0.3$, Fig. $5 \mathrm{~B})$.

PTEN silencing at its maximal efficacy $(72 \mathrm{~h}$ after transfection), induced significant modifications in cell cycle distribution, with first, a decrease in $\mathrm{G}_{0} / \mathrm{G}_{1}$-phase fraction $(\mathrm{P}<0.05)$ from $70.3 \pm 2.7 \%$ in siRNA neg. cells to $66 \pm 2 \%$ in siPTEN cells (Table II). This effect of PTEN silencing on $\mathrm{G}_{0} / \mathrm{G}_{1}$-phase fraction was associated with a significant increase $(\mathrm{P}<0.05)$ in $\mathrm{G}_{2} / \mathrm{M}$-phase fraction from $13.8 \pm 3.5$ to $17.9 \pm 2.6 \%$ whereas $\mathrm{S}$-phase fraction was unaffected. 
A 24-h exposure of siPTEN cells to cetuximab induced a significant and expected increase in $G_{0} / G_{1}$-phase fraction from $66 \pm 2$ to $73.8 \pm 5.2 \%$ (Table II, $\mathrm{P}<0.05$ ) to the same extent than in siRNA neg. cells. This accumulation resulted in a significant depletion in S-phase fraction. Contrarily, the increase in $\mathrm{G}_{2} / \mathrm{M}$-phase fraction observed in siPTEN cells was not influenced by cetuximab exposure (17.9 2.6 vs. $18.2 \pm 1 \%)$.

Taken together, the results achieved in all experiments suggest that Cal 27 cell proliferation and viability do not rely on PTEN status. Consequently, cetuximab effects in Cal 27 cells are not dependent on PTEN expression.

\section{Discussion}

Targeted therapies, such as anti-EGFR monoclonal antibody have been developed to enhance specificity of anti-cancer treatment. In HNSCC samples from patients, EGFR overexpression is a very common event (90-100\%). However, even in case of a strong expression of the molecular target, modest response rates have been observed, especially when cetuximab is used as monotherapy (5-15\% responsive patients) (8). Contrarily to colorectal cancers, detection of KRAS activating mutation in HSNCC as a predictive marker of response to cetuximab therapy seems to be less relevant as these mutations only occur in $\sim 6 \%$ (30). Conversely, constitutive activation of $\mathrm{PI} 3 \mathrm{~K} / \mathrm{AKT}$ pathway is found in $30 \%$ of cases, independently of $K R A S$ status $(26,31,32)$. Based on this, we hypothesized that the reported modest response of HNSCC to cetuximab monotherapy could rely on constitutive activations in PI3K/AKT signaling pathway. Among the mechanisms inducing such activation, PTEN loss of expression has been implicated in several cancer types $(14,16,17,21)$. Recently, Frattini et al (20) have shown that loss of PTEN expression in metastatic colorectal cancer is correlated to cetuximab resistance. To our knowledge, PTEN implication in the response to cetuximab has not been clearly elucidated to date in HNSCC. The aim of our study was to investigate, using siRNA in Cal 27 cell line, the impact of PTEN functionality on cell response to cetuximab.

First, we established that Cal 27 cells were sensitive to cetuximab as signaling pathway activation was substantially inhibited. Cetuximab exposure led to an expected increase in $\mathrm{G}_{0} / \mathrm{G}_{1}$-phase due to its previously described effect of blockade in cell cycle $(33,34)$. Despite this alteration in cell cycle distribution, a modest impact on cell viability and proliferation was found. Additionally and unpredictably, our results indicate that PTEN silencing, led to a drastic reduction in pEGFR expression whereas total EGFR level did not significantly vary.

However, our results confirmed the expected effects of PTEN silencing on the regulation of PI3K/AKT and MAPK signaling pathways, in agreement with already reported data $(35,36)$. Indeed, PTEN silencing in Cal 27 cells led to a constitutive activation of signaling pathways evidenced by a strong increase in pAKT and pERK $1 / 2$ expression, though without inducing any significant promotion of proliferation or cell viability. This downregulation of pEGFR could be related to a negative feedback involving activated ERK 1/2 (37). Taken together, these results suggest that Cal 27 cells are not strictly dependent on EGFR for their viability and proliferation. Further investigations on potential cross talk occurring in Cal 27 cells might be meaningful.

Strikingly, despite this overactivation of signaling pathways ruling cell survival and proliferation in siPTEN cells, cetuximab fully exerts pAKT and pERK 1/2 inhibition of expression, similarly to its effect in untransfected Cal 27 cells. Thus, our study established that in Cal 27 cells, loss of PTEN expression does not lead to a loss of cetuximab efficacy in inhibiting EGFR-downstream signaling pathways, contrarily to data shown in previous works conducted in other tumor types $(14,19-21)$.

In parallel, our results show slight but significant changes in cell cycle distribution of PTEN-silenced cells. A significant decreased in $G_{0} / G_{1}$-phase fraction was observed, suggesting that siPTEN cells entered the $\mathrm{S}$ phase more rapidly. It has been described that PTEN is able to control $\mathrm{G}_{1} / \mathrm{S}$ transition either by PI3K/AKT-dependent or independent effects $(25,38)$, notably through its nuclear localization. Our results are in line with the observations made by Chung et al (39) concerning the PTENmediated growth control through its function in the nucleus. In this study, PTEN has been reported to be able to down-regulate cyclin D1 protein level, inducing a $\mathrm{G}_{0} / \mathrm{G}_{1}$-cell cycle arrest (38).

It is of interest to note that a slight accumulation of siPTEN cells in $\mathrm{G}_{2}$ /M-phase was also observed, without any change in $\mathrm{S}$ phase, additionally to the effects on $\mathrm{G}_{0} / \mathrm{G}_{1}$-phase. Cetuximab exposure did not affect this accumulation of cells in $G_{2} / M$ phase fraction, but kept its ability to block $\mathrm{G}_{1} / \mathrm{S}$ transition regardless of the PTEN status.

Collectively, our results achieved in siPTEN cells, strikingly indicate that cetuximab keeps full ability to inhibit EGFR-dependent mechanisms, as shown by a decreased pAKT and pERK 1/2 level of expression, despite a strong PTEN silencing-induced overactivation. Moreover, siPTEN cells did not show any significant changes either in cell viability or proliferation, only slight modifications on cell cycle. As siRNA silencing was a transient phenomenon, it is likely that the direct impact relying on PTEN loss of expression is a strong and immediate activation of signaling pathways (within 24-48 h), whereas cellular effects like proliferation might be delayed. Indeed, cell cycle analysis revealed slight changes but our experimental set up did not allow observing the expected PTEN silencing effects on cell proliferation. A sustained inhibition by shRNA in Cal 27 cells might provide supplementary data and constitute a more accurate model of PTEN-deficient tumors in vitro.

In conclusion, cetuximab exposure was able to induce a significant reduction in cell viability independently of PTEN expression and kept an effect of inhibition on pAKT and pERK 1/2 despite a PTEN-silencing overactivation of key signaling pathway proteins.

\section{Acknowledgements}

The authors are very grateful to Carole Ramacci and Marie Rouyer in Centre Alexis Vautrin for their helpful technical support. We are very thankful to Professor Philippe Becuwe for his critical review of this manuscript and Dr Stephanie Grandemange for her valuable collaboration. We Thank Dr Patrick Erbacher (PolyPlus Transfection) for providing INTERFERin. This study was supported by grants from the 
French 'Ligue Nationale contre le Cancer' and the 'Region Lorraine'.

\section{References}

1. Jorissen RN, Walker F, Pouliot N, Garett TP, Ward CW and Burgess AW: Epidermal growth factor receptor: mechanisms of activation and signaling. Exp Cell Res 284: 31-35, 2003.

2. Yarden Y: The EGFR family and its ligands in human cancer: signaling mechanisms and therapeutic opportunities. Eur Cancer 4: S3-S8, 2001.

3. Mendelsohn $\mathrm{J}$ and Baselga $\mathrm{J}$ : The EGF receptor family as target for cancer therapy. Oncogene: 19: 6550-6565, 2000.

4. Monnier L, Milano G, Penault-Llorca F, and Merlin JL: Targeting of membrane receptor tyrosine kinases: is there resistance in the HER? Bull Cancer 91: 685-694, 2004

5. Reuter CWM, Morgan MA and Eckardt A: Targeting EGFreceptor-signaling in squamous cell carcinomas of the head and neck. Br J Cancer 96: 408-416, 2007.

6. Ford AC and Grandis JR: Targeting epidermal growth factor receptor in head and neck cancer. Head Neck 25: 67-73, 2003.

7. Harari PM: Epidermal growth factor receptor inhibition strategies in oncology. Endocr Relat Cancer 11: 689-708, 2004.

8. Cohen EEW: Role of epidermal growth factor receptor pathwaytargeted therapy in patients with recurrent and/or metastatic squamous cell carcinoma of the head and neck. J Clin Oncol 24 2659-2665, 2006.

9. Morgillo F, Bareschino MA, Bianco R, Tortora G and Ciardello F: Primary and acquired resistance to anti-EGFR targeted drugs in cancer therapy. Differentiation 75: 788-799, 2007.

10. Lim J, Kim JH, Paeng JY, Kim MJ, Hong SD, Lee JI and Hong SP: Prognostic value of activated Akt expression in oral squamous cell carcinoma. J Clin Pathol 58: 1199-1205, 2005.

11. Yamatodani T, Ekblad L, Kjellen E, Johnsson A, Mineta H and Wennerberg J: EGFR status and persistent activation of Akt and p44/p42 MAPK pathways correlate with the effect of cetuximab in head and neck and colon cancer cell lines. J Cancer Res Clin Oncol 135: 395-402, 2009.

12. Albanell J, Codony-Servat J, Rojo J, Del Campo JM, Sauleda S, Anido J, Raspall G, Giralt J, Rosello J, Nicholson RI, Mendelsohn J and Baselga J: Activated extracellular signalregulated kinases: association with EGFR/TGF $\alpha$ expression in HNSCC and inhibition by anti-EGFR treatments. Cancer Res 61: 6500-6510, 2001.

13. Laurent-Puig P, Cayre A, Manceau G, Buc E, Bachet JB, Lecomte T, Rougier P, Lièvre A, Landi B, Boige V, Ducreux M, Ychou M, Bibeau F, Bouché O, Reid J, Stone S and PenaultLlorca F: Analysis of PTEN, BRAF and EGFR status in determining benefit from cetuximab therapy in wild-type KRAS metastatic colon cancer. J Clin Oncol 27: 5924-5930, 2009

14. Loupakis F, Pollina L, Stasi I, Ruzzo A, Scartozzi M, Santini D, Masi G, Graziano F, Cremolini C, Rulli E, Canestrari E, Funel N, Schiavon G, Petrini I, Magnani M, Tonini G, Campani D, Floriani I and Cascinu S: PTEN expression and KRAS mutations on primary tumors and metastases in the prediction of benefit from cetuximab plus irinotecan for patients with metastatic colorectal cancer. J Clin Oncol 27: 2622-2629, 2009 .

15. Balko JM and Black EP: A gene expression predictor of response to EGFR-targeted therapy stratifies progression-free survival to cetuximab in KRAS wild-type metastatic colorectal cancer. BMC Cancer 9: 145-155, 2009.

16. Janmaat ML, Kruyt FAE, Rodriguez JA and Giaccone G: Response to EGFR inhibitors in NSCLCC: limited antiproiferative effects and absence of apoptosis associated with persistent activity of ERK or AKT kinase pathways. Clin Cancer Res 9: 2316-2326, 2003.

17. She QB, Solit D, Basso A, and Moasser MM: Resistance to gefitinib in PTEN-null HER-overexpressing tumor cells can be overcome through restoration of PTEN function or pharmacologic modulation of constitutive phosphatidylinositol 3'-kinase/Akt pathway signaling. Clin Cancer Res 9: 4340-4346, 2003.

18. Pandolfi PP: Breast Cancer - loss of PTEN predicts resistance to treatment. N Engl J Med 351: 337-2338, 2004.

19. Jhawer M, Goel S, Wilson AJ, Montagna C, Ling YH, Byun DS, Nasser S, Arango D, Shin J, Klampfer L, Augenlicht LH, Perez Soler R and Mariadason JM: PIK3CA mutation/PTEN expression status predicts response of colon cancer cells to the EGFR inhibitor cetuximab. Cancer Res 68: 1953-1961, 2008.
20. Frattini M, Saletti P, Romagnani E, Martin V, Molinari F, Ghisletta M, Camponovo A, Etienne LL, Cavalli F and Mazzucchelli L: PTEN loss of expression predicts cetuximab efficacy in metastatic colorectal cancer patients. Br J Cancer 97: 1139-1145, 2007

21. Bouali S, Chrétien AS, Ramacci C, Rouyer M, Becuwe P and Merlin JL: PTEN expression controls cellular response to cetuximab by mediating PI3K/AKT and RAS/RAFMAPK downstream signaling in KRas wild-type hormone refractory prostate cancer cells. Oncol Rep 21: 731-735, 2009.

22. Liu W, Zhou Y, Reske SN and Shen C: PTEN mutation: many birds with one stone in tumorigenesis. Anticancer Res 28: 3613-3620, 2008

23. Ali IU, Schriml LM and Dean M: Mutational spectra of PTEN/ MMAC1 gene: a tumor suppressor with lipid phosphatase activity. J Natl Cancer Inst 91: 1922-1932, 1999.

24. Gu J, Tamura M and Yamada K: Tumor suppressor PTEN inhibits integrin- and growth factor-mediated MAP kinase signaling pathways. J Cell Biol 143: 1375-1383, 1998.

25. Sansal I and Sellers WR: The biology and clinical relevance of the PTEN tumor suppressor pathway. J Clin Oncol 22: 2954-2963, 2004.

26. Lee JI, Soria JC, Hassan KA, El-Naggar AK, Tang X, Liu DD, Ki Hong W and Mao L: Loss of PTEN expression as a prognostic marker for tongue cancer. Arch Otolaryngol Head Neck Surg 127: 1441-1445, 2001.

27. Squarize CH, Castilho RM and Santos Pinto D: Immunohistochemical evidence of PTEN in oral squamous cell carcinoma and its correlation with the histological malignancy grading system. J Oral Pathol Med 7: 379-384, 2002

28. Schimanski CC, Linnemann U and Berger MR: Sensitive detection of K-ras mutations augments diagnosis of colorectal cancer metastases in the liver. Cancer Res 59: 5169-5175, 1999.

29. Chergui F, Chrétien AS, Bouali S, Ramacci C, Rouyer M, Bastogne T, Genin P, Leroux A and Merlin JL: Validation of a phosphoprotein array assay for characterization of human tyrosine kinase receptor downstream signaling in breast cancer. Clin Chem 55: 1327-1336, 2009.

30. Weber A, Langhanki L, Sommerer S, Markwarth A, Wittekind C and Tannapfel A: Mutations of the BRAF gene in squamous cell carcinoma of the head and neck. Oncogene 22: 4757-4759, 2003.

31. Massarelli E, Liu DD, Lee JJ, El-Naggar AK, Lo Muzio L, Staibano S, De Placido S, Myers JN and Papadimitrakopoulou VA: Akt activation correlates with adverse outcome in tongue cancer. Cancer 104: 2430-2436, 2005.

32. Pedrero JMG, Carracedo DG, Munoz Pinto C, Zapatero AH, Rodrigo JP, Nieto CS and Gonzalez MV: Frequent genetic and biochemical alterations of the PI3-K/AKT/PTEN pathway in head and neck squamous cell carcinoma. Int J Cancer 114: 244-248, 2005.

33. Kiyota A, Shintani S, Mihara M, Nakahara Y, Ueyama Y, Matsumara T, Tachikawa T and Wong DT: Anti-epiderml growth factor receptor monoclonal antibody 225 upregulates $\mathrm{p} 27^{(\mathrm{Kip} 1)}$ and $\mathrm{p} 15^{\text {(Ink4B) }}$ and induces G1 arrest in oral squamous carcinoma cell lines. Oncology 63: 92-98, 2002.

34. Harari PM and Huang SM: Head and neck cancer as a clinical model for molecular targeting of therapy: combining EGFR blockade with radiation. Int J Radiat Oncol Biol Phys 49: 427-433, 2001.

35. Maehama T: PTEN: its deregulation and tumorigenesis. Biol Pharm Bull 30: 1624-1627, 2007.

36. Vitolo MI, Weiss MB, Szmacinski M, Tahir K, Waldman T, Ho Park B, Martin SS, Weber DJ and Bachman KE: Deletion of PTEN promotes tumorigenic signaling, resistance to anoikis, and altered response to chemotherapeutic agents in human mammary epithelial cells. Cancer Res 69: 8275-8283, 2009.

37. Hayashi H, Tsuchiya Y, Nakayama K, Satoh T and Nishida E: Down-regulation of the PI3-kinase/AKT pathway by ERK MAP Kinase in growth factor signaling. Genes Cells 13: 941-947, 2008.

38. Weng LP, Brown JL and Eng C: PTEN induces apoptosis and cell cycle arrest through PI3K/AKT-dependent and independent pathways. Hum Mol Genet 10: 237-242, 2001.

39. Chung JH, Ostrowski MC, Roomigh T, Minaguchi T, Waite KA and Eng C: The ERK1/2 pathway modulates nuclear PTENmediated cell cycle arrest by cyclin D1 transcriptional regulation. Hum Mol Genet 17: 2553-2559, 2006. 